\title{
DESIGN OF CHAOTIC BIFURCATION PARAMETER MODULATION SCHEME USING MEAN VALUE ESTIMATION
}

\author{
Hikmat N. Abdullah ${ }^{1}$, Ibrahim F. Ali ${ }^{2}$ \\ ${ }^{1}$ College of Information Engineering, Al-Nahrain University, Iraq \\ ${ }^{2}$ Electrical Engineering Department, Al-Mustansiryah University, Iraq \\ dr.h.abdullah@ieee.org ${ }^{1}$, ibraheem_1989@yahoo.com ${ }^{2}$ \\ (Received: 27/01/2018; Accepted: 17/03/2018)
}

\begin{abstract}
Chaotic modulation schemes has proved to be an efficient wideband modulation schemes due it its comparable performance to spread spectrum systems. The ergodic chaotic parameter modulation scheme (ECPM) is one of such schemes. However, the conventional ECPM uses sophisticated techniques to estimate the bifurcation parameter which increase the demodulator complexity. In this paper, a mean value estimation technique is proposed for use at the receiver for retrieving the original information to minimize the computational complexity. Binary Phase Shift Keying Ergodic Chaotic Parameter Modulation (BPSK-ECPM) scheme is used to modulate the information signal in the bifurcating parameter of the chaotic map. Simulation results showed that besides the proposed scheme simplifies the demodulator structure, it also improves bit error rate (BER). For instance, in AWGN channel and at of $10^{-3}$, BPSK-ECPM achieves gains in $E_{b} / N_{0}$ of $0.7 \mathrm{~dB}$ and $5 \mathrm{~dB}$ in comparison with conventional direct sequence spread spectrum with BPSK modulation (DS-SS BPSK) and conventional ergodic chaotic parameter modulation spread spectrum (ECPM-SS) systems respectively. The results also confirm that in Rayleigh fading channel and at BER of $10^{-3}$, BPSK-ECPM achieves gains in $E_{b} / N_{0}$ of about 6.3dB and 1.6dB in comparison with DS-SS BPSK and ECPM-SS systems respectively.
\end{abstract}

Keywords - Chaotic modulation, Ergodic theory, Parameter modulation, Mean value estimation.

\section{INTRODUCTION}

Recently, applications of chaotic signals in communication systems got a great attention. Many active ongoing researches have been looking at ways to exploit the properties of chaotic signals in chaotic communication schemes and have got a quite remarkable results. Chaotic signals offer many advantages to spread spectrum (SS) communications such as utilizing wide bandwidth, having low power spectral density, pseudo-randomness, noise-like, robustness in multipath environments, resistance to jamming, ergodicity, long term unpredictability, non-periodic and difficult to predict and to reconstruct. Furthermore, chaotic signals are easily generated using simple hardware implementation; thereby, reducing the manufacturing cost. The above mentioned characteristic of chaotic signals make it attractive for communication use since it offers good and cheaper substitution to conventional SS communication systems [1,2].

Different chaotic modulation systems have been presented to exploit different properties of a chaotic dynamical system to obtain the aim of SS transmission [3-8]. One of the most important chaotic modulation systems is chaotic parameter modulation (CPM) schemes [4].

In CPM schemes, the information signal is modulated by merging it into the chaotic map parameters to achieve the aim of SS transmission for both analog and digital communication. The chaotic signal parameters that are usually need to be estimated are the bifurcation factor and the initial condition. CPM schemes offer several benefits in comparison with traditional SS systems based on spreading codes. CPM systems does not require synchronization process for data demodulation and has potential of higher system capacity compared to the traditional SS techniques. 
However, the problem in CPM systems is how to make an accurate parameter estimation at the receiver thereby retrieving the transmitted information signal in an optimal manner.

Parameter estimation techniques are most commonly used in chaotic systems for demodulating the information signal. Different adapting filtering techniques based on the different chaotic systems used and different conditions such as least mean square (LMS), improved least squares (ILS), recursive least square (RLS) and extended Kalman filter (EKF) have been developed to estimate and track the parameters of chaotic signals [9-11]. The tracking and estimation capabilities of these techniques made them suitable for parameter estimation problems and demodulating the information signal in a real time communication system. The above adaptive filtering estimation techniques are found not able to guarantee a reliable communication when measurement noise is found [4].

In this paper, an improved technique called mean value estimation for estimating bifurcation parameter of chaotic map is introduced. It is developed such that it combines the simplicity of estimator structure and ability to make accurate estimation in high-level noise situations.

\section{ERGODIC PROPERTIES OF CHAOTIC SYSTEMS}

Ergodic theory is a field of mathematics in which the dynamical systems with an invariant measure and related problems are studied. More accurate information have been provided by different ergodic theorems which state that: under certain conditions, the time average of a function along the trajectories exists almost everywhere and is related to the space average. For the special class of ergodic systems, this time average is the same for almost all initial points. It means that, the initial state has been forgotten for the long time evolving systems [12]. Ergodic theory of chaotic systems deals with the statistical properties of trajectories of dynamical systems. For chaotic dynamical systems, the long- term predictions are almost impossible from initial observations with limited accuracy. The important result in ergodic theory is known as Birkhoff 's point wise ergodic theorem. For each $\theta \epsilon\left[\theta_{\min }, \theta_{\max }\right]$, the chaotic map $\mathrm{f}_{\theta}$ has a unique invariant ergodic measure $\mu_{\theta}$. The chaotic signal $x(t)$ is basically an orbit of $f_{\theta}$ with the starting point $x(0)$.

Based on the Birkhoff ergodic theorem, the limit $\lim _{N \rightarrow \infty} \frac{1}{N} \sum_{i=0}^{N-1} x(i)$ exists and is equal to the constant $\int x d_{\mu_{\theta}}(x)$, this limit is called the mean value function $M(\theta)$ of the chaotic map $f_{\theta}$ and $x(t)$ is the chaotic signal. It is clear that the initial condition $\mathrm{x}(0)$ does not effect in this limit but only the bifurcation parameter $\theta$. For several chaotic $\operatorname{signals,} M(\theta)$ is a monotone function over wide bifurcating parameter range. Hence, the bifurcation parameter can be estimated accurately based on estimation of the mean values of the received signal.

The use of BPSK modulation together with ECPM based on mean value estimation would produce a system with useful features. It does not involve any channel estimation and channel equalization except the estimation of the mean value of the received signal and it can be built by a simple hardware with very low manufacturing cost as compared with conventional correlator for SS communication. The mean value estimator consists of several additions and one multiplication when digital signals is used, and an integrator for analog implementation without any synchronization process. Furthermore, BPSK-ECPM scheme offers robust way of mitigating the multipath fading since the multipath interference effect is a simple scaling factor of the mean value of the received signal and outperformed the conventional DS-SS system for a wide range of channel estimation errors. Therefore, the BPSK-ECPM scheme can 
be a good candidate for the real time wireless communication applications like road to vehicle and vehicle to vehicle data broadcasting in intelligent transportation system.

\section{DESIGN OF BPSK-ECPM SYSTEM}

In this section, the design of BPSK-ECPM scheme transmitter and receiver is given. Depending on the ergodic properties of the chaotic dynamical system and BPSK modulation, the bifurcating parameters values are estimated using mean value estimation and then the original data information are retrieved by a non-coherent detection.

\section{A. The Transmitter Design}

The information data have been modulated into the modulator of BPSK-ECPM scheme by varying the parameters of chaotic system. only one chaotic parameter $\theta_{0}$ is used instead of transition between two bifurcation parameters $\theta_{\mathrm{i}}$, $\mathrm{i}=0,1$ as in conventional ECPM-SS system. The mean value function $\left|\mathrm{M}\left(\theta_{0}\right)\right|$ is assumed to have the largest value within the chaotic regime, that is: $\left|\mathrm{M}\left(\theta_{0}\right)\right|=\max \left|\mathrm{M}\left(\theta_{\mathrm{i}}\right)\right|$ for each $\theta_{\mathrm{i}} \epsilon\left[\theta_{\min }, \theta_{\max }\right]$. After the chaotic parameter is fixed in the chaotic system, BPSK is applied as the RF modulation, as shown in Figure (1). The modulation process can be expressed as:

$$
x^{\prime}(t)= \begin{cases}x(t) & \text { when } s(t)=1 \\ -x(t) & \text { when } s(t)=0\end{cases}
$$

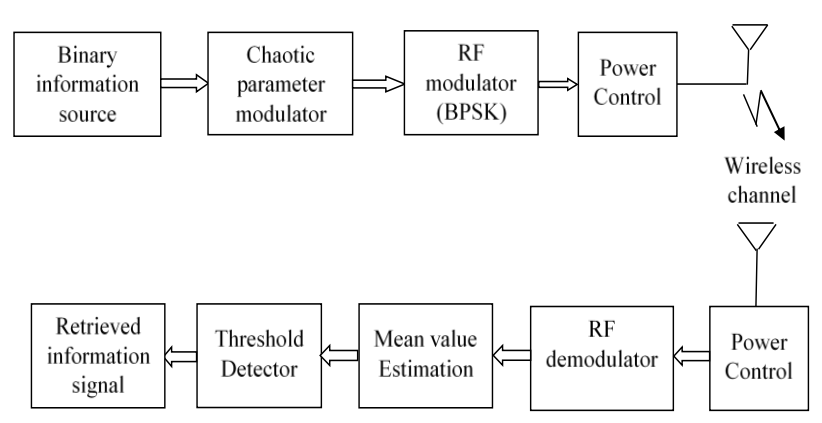

Figure 1. Block diagram of BPSK-ECPM system

The output modulated signal $x^{\prime}(t)$ from a chaos system has a wideband characteristics which is converted up to the RF modulator for spread spectrum (SS) transmission.

\section{B. Design of BPSK-ECPM Receiver}

At the receiver side, the received signal is observed after the radio frequency $(\mathrm{RF})$ demodulator, the received signal corrupted with AWGN signal is $r(t)=x(t)+n(t)$. This signal is then passed to BPSK-ECPM demodulator containing an estimation of the mean value of the received signal and a threshold detector. $x(t)$ represents the chaotic waveform produced from a chaotic system with parameter $\theta_{0}$ and $\mathrm{x}^{\prime}(\mathrm{t})$ represents the chaotic signal after chaotic modulator. Therefore, for transmitting data " 1 ", the chaotic signal $x(t)$ is used, and for transmitting data " $0 "$, the chaotic signal $\mathrm{x}(\mathrm{t})$ is used. Therefore, in BPSK-ECPM, the binary information signal is demodulated by estimating the mean value of the received signal (estimate the first order statistics). Hence, the main difference between the BPSK-ECPM scheme and conventional chaos based SS schemes is that the conventional techniques utilize the signal energy/power (second 
order statistics) for data demodulation. Hence, the design of the receiver must be optimized in order to reduce the effects of noise at the receiver output. Therefore, the chaotic parameter must be estimated accurately for perfect data demodulation. At the receiver, the output of the demodulator after mean value estimation can be expressed as:

$$
\begin{gathered}
M_{k}=\frac{1}{T_{b}} \int_{k T_{b}}^{(k+1) T_{b}} r(t) d t \\
=\mp \frac{1}{T_{b}} \int_{k T_{b}}^{(k+1) T_{b}} x(t) d t+\int_{k T_{b}}^{(k+1) T_{b}} n(t) d t
\end{gathered}
$$

As the expectation of an AWGN is zero, if $\mathrm{T}_{\mathrm{b}}$ is large enough, the term $\frac{1}{T_{b}} \int_{k T_{b}}^{(k+1) T_{b}} x(t) \approx 0$. Therefore, the mean value of the chaotic signal is a constant for different parameters, that is $\frac{1}{T_{b}} \int_{k T_{b}}^{(k+1) T_{b}} x(t) \approx M\left(\theta_{i}\right)$. Hence, the output of the estimation process is $\mathrm{M}_{\mathrm{k}}=\mathrm{M}\left(\theta_{\mathrm{i}}\right)$. Since only one parameter is used to generate the chaotic sequence, the output signal can be simply expressed by $M_{k} \approx \mp M(\theta)$. Since $M\left(\theta_{0}\right)>0$, the decision can be made by setting the threshold detector to zero,

$$
\hat{s}(t)= \begin{cases}0 & \text { when } M_{k}<0 \\ 1 & \text { when } M_{k} \geq 0\end{cases}
$$

Therefore, the threshold is chosen with zero at the detector output value in BPSK-ECPM system. The distance of the mean value obtained from BPSK-ECPM system at the demodulator output is $\Delta M=2\left|\mathrm{M}\left(\theta_{0}\right)\right|$, which is the maximum possible value. Hence, a larger mean value distance can be achieved from BPSK-ECPM system in comparison with conventional ECPM-SS system, which will improve the communication performance.

\section{Simulation RESUlTS OF BPSK-ECPM SYSTEM}

In this section, the probability of error for BPSK-ECPM system with binary modulation in comparison with different conventional SS and chaotic modulation systems in the presence of AWGN and multipath fading channels is presented. For AWGN channel case, the corrupting noise is assumed with zero mean, the variance is $\sigma_{n}^{2}$ and the noise PSD is $N_{0} / 2$. The results that show the effects of synchronization error, high transmission data rate, and the recovery performance of the image signal in ITS system are also presented.

\section{A. Simulation Results in AWGN Channel}

Figure (2) shows the BER performance of BPSK-ECPM system in comparison with conventional DS-SS with BPSK modulation. As shown in Figure (2), at BER $=10^{-3}$, BPSK-ECPM scheme achieves a gain in $\mathrm{E}_{\mathrm{b}} / \mathrm{N}_{0}$ of about 0.7 $\mathrm{dB}$ as compared with conventional DS-SS BPSK system. Hence, BER performance of the BPSK-ECPM scheme outperforms DS-SS BPSK system. BPSK-ECPM utilizes the mean value estimation, while DS-SS BPSK system uses perfect channel estimation. As described above, the ergodic approach can be improved by combining it with the standard BPSK modulation which results in BPSK-ECPM system model. Hence, the system operation is simplified 
and the performance will be improved since only one chaotic parameter is used instead of switching between two chaotic parameters $\theta_{\mathrm{i}}, \mathrm{i}=0,1$, as in ECPM-SS system.

Figure (3) shows the simulation results of BPSK-ECPM in comparison with ECPM-SS system in AWGN channel. In this figure it can be seen that at $\mathrm{BER}=10^{-3}$, BPSK-ECPM scheme achieves a gain in $\mathrm{E}_{\mathrm{b}} / \mathrm{N}_{0}$ of about $5 \mathrm{~dB}$ as compared with ECPM-SS system. Hence, BPSK-ECPM has a better performance than ECPM-SS system in AWGN channel. The BER performance of the BPSK-ECPM system in comparison with conventional chaos based SS digital communication systems is evaluated in AWGN channel.

Figure (4) shows a graph of bit error rate (BER) as a function to $\mathrm{E}_{\mathrm{b}} / \mathrm{N}_{0}$ for some chaos based SS communication schemes including BPSK- ECPM. It is clear in this figure that, at BER $=10^{-3}$, BPSK-ECPM achieves gains in $\mathrm{E}_{\mathrm{b}} / \mathrm{N}_{0}$ of about $1 \mathrm{~dB}, 1.5 \mathrm{~dB}, 5.2 \mathrm{~dB}, 6.5 \mathrm{~dB}, 9 \mathrm{~dB}, 10 \mathrm{~dB}$ and $11 \mathrm{~dB}$, as compared with CSS, CC, FM-DCSK, DCSK, CSK, COOK and QCSK schemes respectively. Hence, BPSK-ECPM has a better performance, as compared with other chaos modulation schemes in AWGN channel.

\section{B. Simulation Results in Multipath Fading Channel}

In this section, the probability of error for BPSK-ECPM system with binary modulation in comparison with different conventional SS and chaotic modulation systems in multipath fading channel with AWGN process is presented. The BER performance of BPSK-ECPM and DS-SS systems in frequency selective fading channel is shown in Figure (5). The plot for DS-SS shows the BER performance using imperfect channel estimation with maximum likelihood (ML) estimator, while the BPSK-ECPM utilizes the mean value estimator only without channel estimation and channel equalization. As shown in Figure (5), it is clear that, at BER=10-3, BPSK-ECPM scheme achieves a gain in $\mathrm{Eb} / \mathrm{N} 0$ of about $6.3 \mathrm{~dB}$, as compared with DS-SS system. Hence, BPSK-ECPM has a better performance than DSSS system in frequency selective fading channel. Figure (6) shows the simulation results of BPSK-ECPM in comparison with ECPM-SS systems in frequency selective fading channel. It can be seen in this figure that, at $\mathrm{BER}=10-3$, BPSK-ECPM scheme achieves a gain in Eb/N0 of about1.6dB, as compared with ECPM-SS system. Hence, BPSK-ECPM has a slightly better performance than ECPM-SS system in frequency selective fading channel.

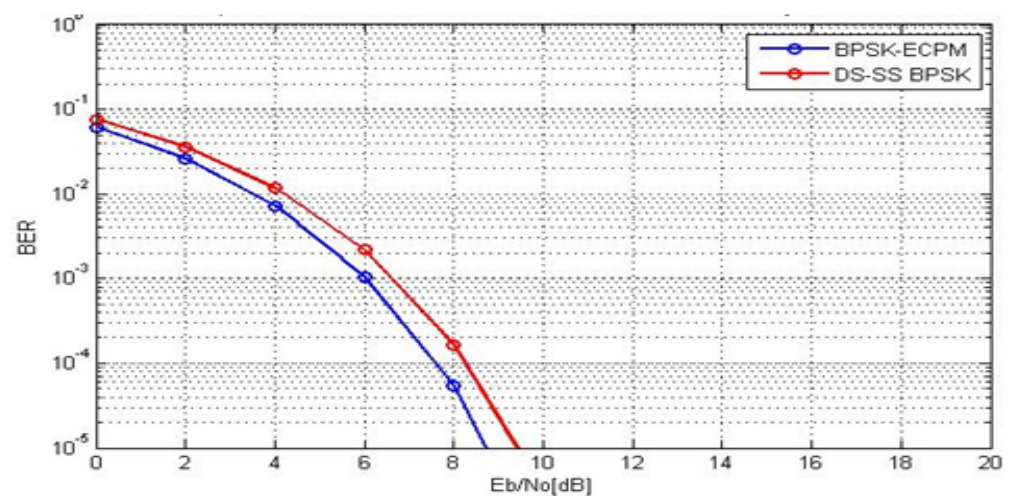

Figure 2. BER performance comparison of conventional DS-SS BPSK and BPSK-ECPM systems in an AWGN channel. 


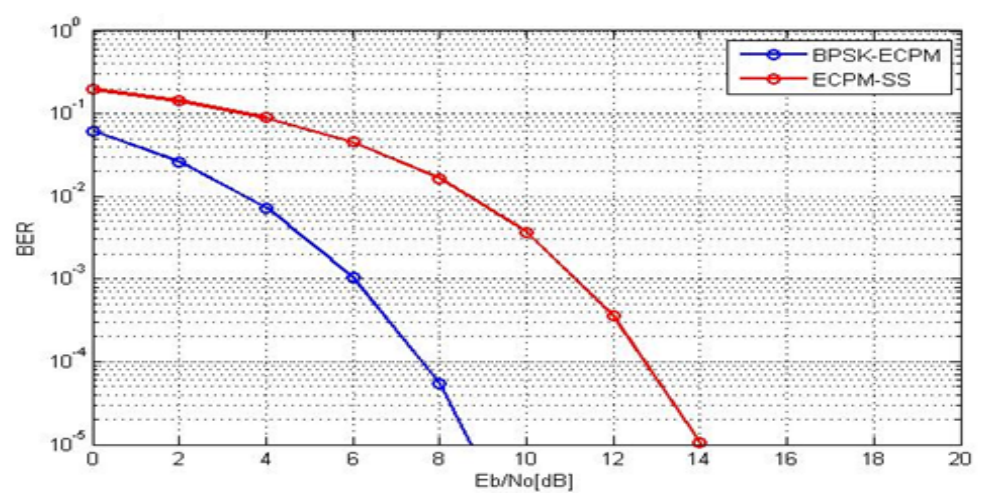

Figure 3. Performance of BPSK-ECPM in comparison with ECPM-SS in AWGN channel.

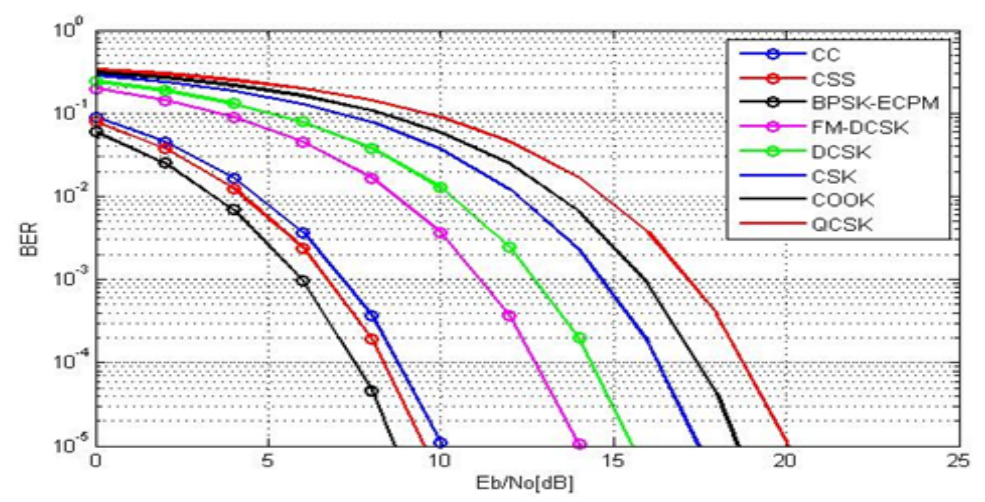

Figure 4. BER performance of different chaos based schemes in AWGN channel.

The BER performances of all chaos based SS systems including BPSK-ECPM system are evaluated through frequency selective fading channel, as shown in Figure (7). It is clear that, the chaos based communication systems: CSK, COOK and QCSK fail to operate normally in Rayleigh fading channel and their BER performances are deteriorated. The demodulators of these systems cannot handle the large amount of noise thereby fail to retrieve the transmitted data information accurately. For CC and CSS, the reference signal is used in order to perform the correlation with the received signal. Hence, the unwanted signals are removed in the demodulation process by means of the correlator. Therefore, the transmitted data can be retrieved accurately. At BER $=10^{-3}$, CSS scheme achieves gain in $\mathrm{E}_{\mathrm{b}} / \mathrm{N}_{0}$ of about $0.7 \mathrm{~dB}$ over $\mathrm{CC}$ scheme. For FM-DCSK, the performance under multipath channel has been evaluated. As FM-DCSK is a technique which combines the modulation with a spread spectrum (SS) property. Hence, it is an effective technique that can achieve an excellent anti-multipath fading capability. At the receiver end, a selfsynchronized correlator is employed which makes the demodulation process continues to operate properly even though the time delay $\tau$ causes the correlation process shift by some time interval. From Figure (7), at BER=10 ${ }^{-3}$, FM-DCSK achieves gain in $\mathrm{E}_{\mathrm{b}} / \mathrm{N}_{0}$ of about $7 \mathrm{~dB}$ compared to DCSK scheme which also uses a self-synchronized correlator at the receiver. Finally, the performance of BPSK-ECPM in a multipath channel is evaluated. BPSK-ECPM scheme is robust to multipath fading effect. The operation of BPSK-ECPM system is still well under multipath channel since the effect of the multipath signals is a simple scaling factor of the mean value of the received signal. At BER=10 ${ }^{-3}$, BPSK-ECPM scheme achieves gains in $\mathrm{E}_{\mathrm{b}} / \mathrm{N}_{0}$ of about $4.8 \mathrm{~dB}, 5.8 \mathrm{~dB}, 6.5 \mathrm{~dB}$ and $11.8 \mathrm{~dB}$, as compared with FM-DCSK, CSS, CC 
and DCSK schemes respectively. Hence, BPSK-ECPM has a better performance, as compared with other chaos modulation schemes in a multipath fading channel.

\section{Simulation Results under the Effect of Synchronization Error}

In communication systems, the term synchronization refers to the requirement of generation a local carrier reference at the receiver which is exact replica to that at the transmitter. The demodulator; therefore, has a coherent detection. If the transmitted signal is subjected to a time delay $\tau$, the communication system will operate under synchronization error. When a coherent communication system operates without perfect synchronization, or when there is an error in the synchronization process, it will be a disaster to this communication system. The maximum value of the synchronization error is $T_{b} / 2$, where $T_{b}$ is the bit duration and the propagation delay $\tau$ is a small fraction of $\mathrm{T}_{\mathrm{b}}$, about $\mp 1 \%$ of $\mathrm{T}_{\mathrm{b}}$ in a practical application.

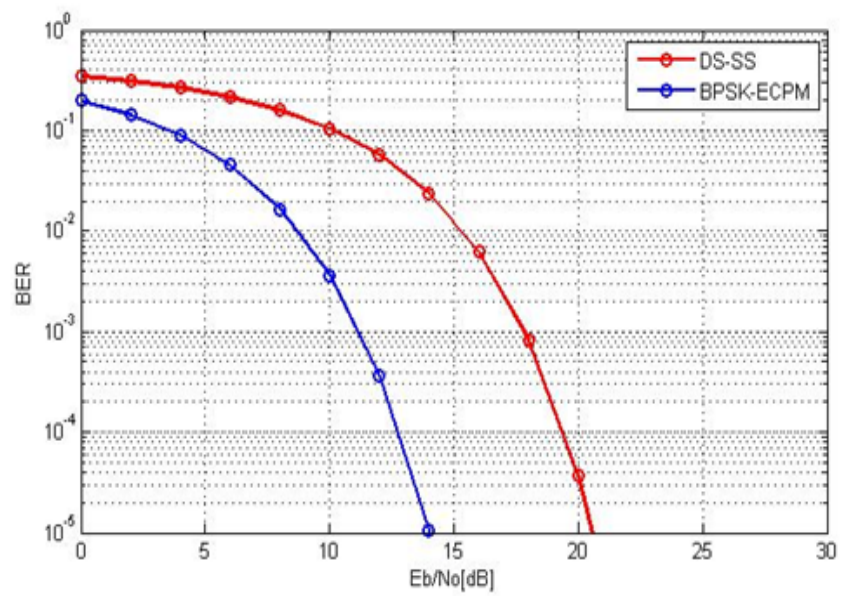

Figure 5. BER performance of BPSK-ECPM in comparison with DS-SS in frequency selective fading channel.

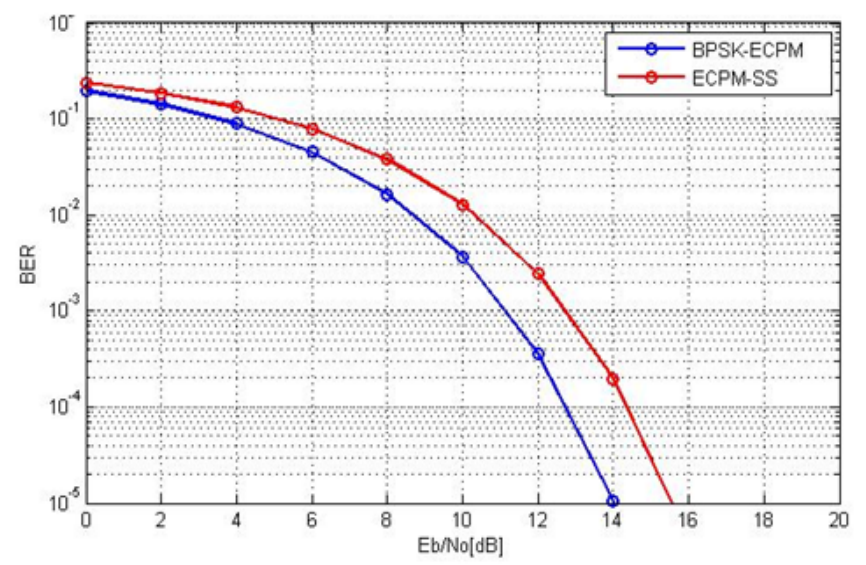

Figure 6. BER performance of BPSK-ECPM in comparison with ECPM-SS in frequency selective fading channel. 


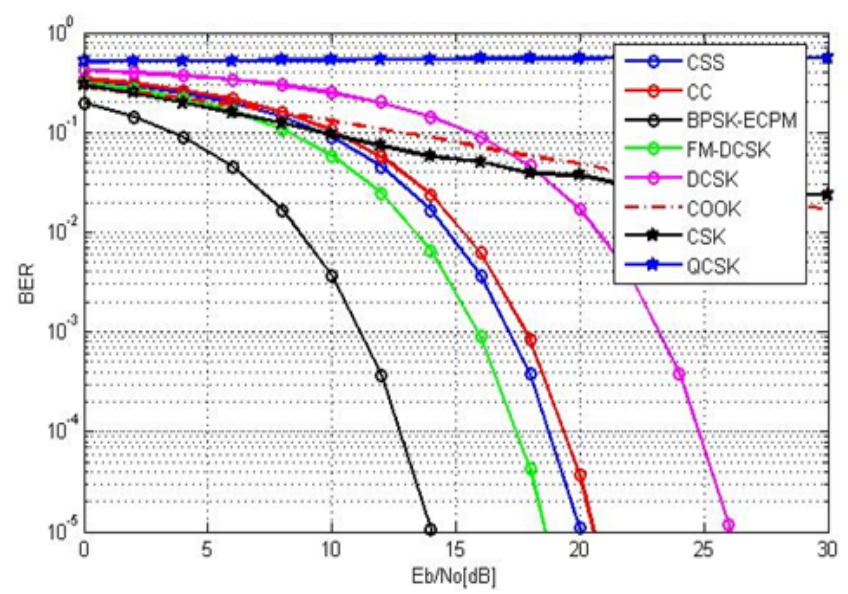

Figure 7. BER performance of the different chaos based schemes in frequency selective channel.

The BER performances of all chaos based communication systems are evaluated under synchronization error as shown in Figure (8). From Figure (8), the BER performances of different chaos based schemes considered are not the same. For a coherent chaos based schemes such as CSS, CC and CSK, the time delay $\tau$ makes the output of the correlation process between the received signal and the reference signal is equal to zero. Therefore, their demodulators fail to retrieve the transmitted information signal accurately when these communication systems lose synchronization. For DCSK and FM-DCSK systems, a self-synchronized correlator can achieve an ability to make their demodulators still operate properly, but at the expense of increasing the $E_{b} / N_{0}$ level. Since the propagation delay $\tau$ is distributed within the interval $\left[0, \mathrm{~T}_{b} / 2\right]$ and the self-correlation is based within each half bit duration $\mathrm{T}_{\mathrm{b}} / 2$, the BER performance of DCSK and FM-DCSK depends on the value of the delay $\tau$, and the big value of $\tau$ results in worse BER performance and vice versa. At $B E R=10^{-3}$, FM-DCSK achieves gain in $\mathrm{E}_{\mathrm{b}} / \mathrm{N}_{0}$ of about $2 \mathrm{~dB}$ in comparison with DCSK system.

For a general chaotic parameter modulation (CPM) schemes, the adaptive filtering algorithms can estimate and track the chaotic parameters without any synchronization process. The propagation delay $\tau$ does not cause a much error to their demodulators. Therefore, the BER performances of these schemes remain unchanged even for large value of time delay $\tau$. Therefore, the operation of BPSK-ECPM system is still the same as its operation in the multipath channel where the synchronization error does not affect its performance. From Figure (8), it can be seen that, at $\mathrm{BER}=10^{-3}$, BPSK-ECPM achieves gains in $\mathrm{E}_{\mathrm{b}} / \mathrm{N}_{0}$ of about $4.8 \mathrm{~dB}$ and $6.8 \mathrm{~dB}$, as compared with FM-DCSK and DCSK schemes respectively. Hence, BPSK-ECPM has a better performance as compared with other chaos modulation schemes under the effect of synchronization error.

\section{Simulation Results under the Effect of Higher Transmission Data Rate.}

One of the most important factors that affects the BER performance for these chaos based communication systems is the high transmission data rate. The BER performance of all chaos based schemes is evaluated here under this condition. In this simulation, the data rate is fixed at $1 \mathrm{Mbps}$ as it is the speed used in cable communication and wireless local area network (WLAN). Figure (9) shows the BER performance of different chaos based schemes under 
high transmission data rate in AWGN channel. As shown in this figure CSK scheme fails to operate normally under high transmission data rate. DCSK system can operate well under high transmission data rate only if the bit duration is large enough. Otherwise, the DCSK system will suffer from the estimation problem and its decision is based on signal variance and affected by the noise variance. Therefore, the demodulator for this system is related to the bit duration $T_{b}$. Hence, they can operate well only when the bit duration $T_{b}$ is large enough, because when the bit duration $\mathrm{T}_{\mathrm{b}}$ is decreased, in other words, when the data rate is increased, the variance also increases which results in a larger decision error. In DCSK system BER $=10^{-3}$ can be achieved at $\mathrm{E}_{\mathrm{b}} / \mathrm{N}_{0}$ of about $13.7 \mathrm{~dB}$. For CSS, CC and FM-DCSK systems, the BER performances of these schemes are not really affected when high transmission data rate is employed. Therefore, if the energy per bit is fixed in these systems, the higher data rate has a little effect on the operation of the demodulators of these systems. At $\mathrm{BER}=10^{-3}$, the $\mathrm{E}_{\mathrm{b}} / \mathrm{N}_{0}$ level is about $6.7 \mathrm{~dB}, 7.2 \mathrm{~dB}$ and $12.2 \mathrm{~dB}$ for $\mathrm{CSS}, \mathrm{CC}$ and FM-DCSK systems respectively. General chaotic parameter modulation (CPM) schemes take the advantage of being unrelated with the bit duration. Therefore, the data rate for CPM system could be higher than other chaotic modulation systems. Finally, the BER performance for BPSK-ECPM system under high transmission data rate is evaluated. Simulation results show that, at $\mathrm{BER}=10^{-3}$, BPSK-ECPM achieves gains in $\mathrm{E}_{\mathrm{b}} / \mathrm{N}_{0}$ of about $0.7 \mathrm{~dB}, 1.2 \mathrm{~dB}, 6.2 \mathrm{~dB}$ and $7.7 \mathrm{~dB}$, as compared with CSS, CC, FM-DCSK and DCSK schemes respectively. Therefore, BPSK-ECPM system has a better performance than all chaos based communication systems when high data transmission rate is employed.

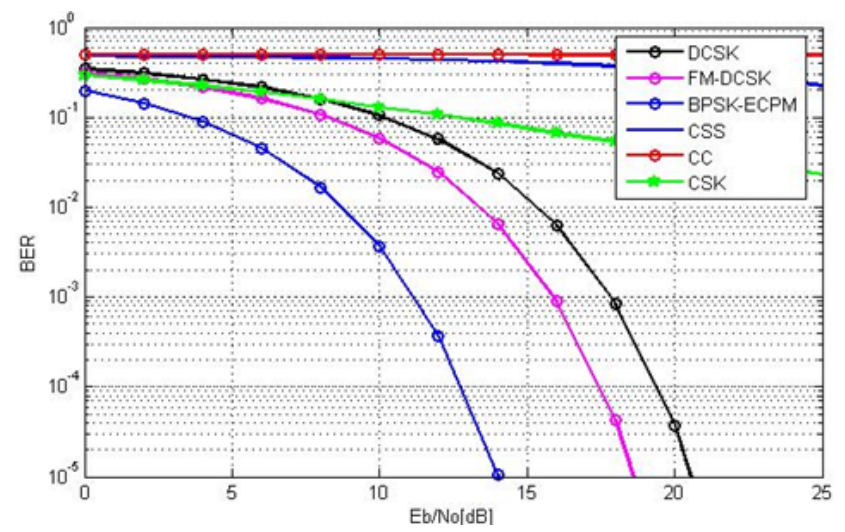

Figure 8. Effect of synchronization error on the different chaos based communication schemes.

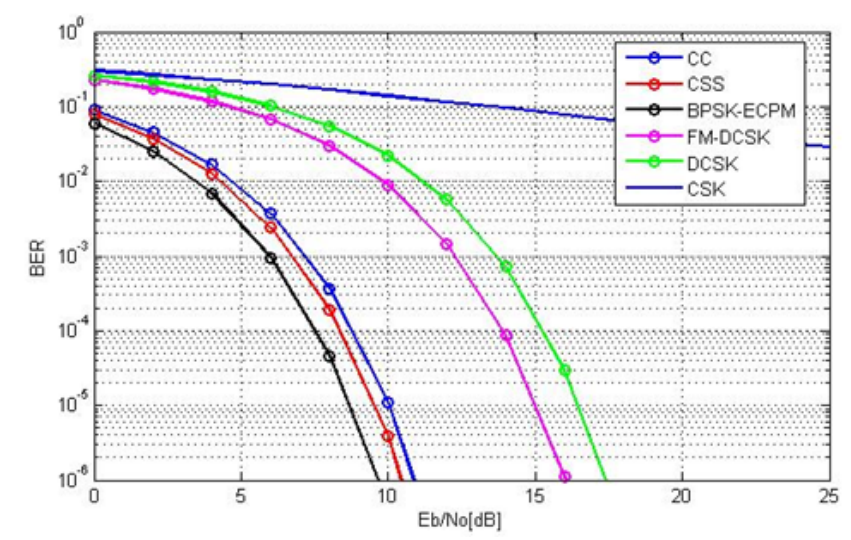

Figure 9. Effect of high transmission data rate on the different chaos based communication schemes 


\section{CONCLUSIONS}

Using ergodic properties of chaotic signals, a mean value estimation technique is proposed for use at the receiver of BPSK-ECPM system in order to retrieve the bifurcating parameter and hence determining the transmitted original information. Non-coherent detection is used in this technique to eliminate the synchronization problem; thereby, reduces the complexity of the demodulator. Therefore, BPSK-ECPM scheme is an ideal candidate for the data broadcasting in real time wireless applications. The main drawback of the mean value estimation technique is that, it cannot be applied to any chaotic system in general. Only the chaotic maps that have a monotone mean value function. BPSK-ECPM scheme offers improved performance over conventional DS-SS system with BPSK modulation, conventional ECPM and conventional chaos modulation schemes: CSS, CC, CSK, COOK, QCSK, DCSK and FMDCSK in AWGN and multipath fading channels. Furthermore, BPSK-ECPM scheme has improved performance over the conventional chaos based modulation systems under the effect of synchronization error and high transmission data rate problems.

\section{REFERENCES}

[1] Hikmat N. Abdullah, Saad S. Hreshee and Ameer K. Jawad, "Design of Efficient Noise Reduction Scheme for Secure Speech Masked by Chaotic Signals”, Journal of American Science, Vol.11, No.7, pp. 49-55, May 2015.

[2] G. Kaddoum, A. Lawrance, P. Chargé and D. Roviras, "Chaos Communication Performance: Theory and Computation", Journal of Circuits, Systems, and Signal Processing, Springer Science and Business Media, Volume 30, Issue 1, pp. 185-208,.February 2011.

[3] Nguyen Xuan Quyen, Ngyyen Tung Lam, Do Huy Duy, Phong Nguyen and Nguyen-Son Vo, "Chaos-based spread-spectrum using M-ary PSK and OFDM-MIMO", Proceedings of International Conference on Signal Processing, Telecommunications and Computing SigTelCom2017, Da Nang, Vietnam, 9-11 January 2017.

[4] Jianbin He and Jianping Cai, "Parameter modulation for secure communication via the synchronization of Chen hyperchaotic systems", Journal of Systems Science \& Control Engineering, Taylor and Francis Publications, Vol.2, Issue1, pp.718-726, 2014.

[5] Nguyen Xuan Quyen, Vu Van Yem, and Thang Manh Hoang, " A Chaos-Based Secure Direct-Sequence/SpreadSpectrum Communication System", Journal of Abstract and Applied Analysis, Hindawi Publishing Corporation, Volume 2013.

[6] Hikmat N. Abdullah and Ali A. Radhi, "Chaotic Multiple Access System Based on Orthogonal Chaotic Vector of Lorenz System", Al-Nahrain University College of Engineering Journal NUCEJ, Vol.18, No.2, pp.219-228, December 2015,

[7] Leonard Novosel and GordanŠišul, "Performance evaluation of chaotic spreading sequences on software-defined radio", EURASIP Journal on Wireless Communications and Networking, Vol.80, pp. 1-12, 2017.

[8] Hikmat N. Abdullah and Ibrahim F. Ali, "Modified Maximum Auto-Correlation Estimation for Chaotic Initial Condition Modulation Scheme, "Engineering and Development Journal, Vol.20, No.1, pp.84-89, January 2016.

[9] Inés P. Mariño, Ekkehard Ullner, and Alexey Zaikin, "Parameter Estimation Methods for Chaotic Intercellular Networks", PLoS One Journal, Vol.8, Issue 11, 2013. doi: 10.1371/journal.pone.0079892

[10] Hao Li, Peng Bai, Jun-Jie, Jie Zhu and Hui Zhang, "Parameter Estimation of Chaotic Systems Using Fireworks Algorithm", International Conference in Swarm Intelligence ICSI, Beijing, China, 25-28 June 2015.

[11]J. Hakkarainen, A. Ilin, A. Solonen, M. Laine, H. Haario, J. Tamminen, E. Oja, and H. J'arvinen, “On closure parameter estimation in chaotic systems", Journal of Nonlinear Processes in Geophysics, Vol.19, pp.127-143, 2012.

[12]Henry Leung, S. Shanmugam, Nan Xie, and Schun Wang, "An ergodic approach for chaotic signal estimation at low SNR with application to ultra-wide-band communication”, Journal IEEE Transactions on Signal Processing, Vol.54 Issue 3, pp.1091-1103, March 2006. 